\title{
The frequency and the arterial branches of the colosigmoid arterial trunk
}

Department of Anatomy, Faculty of medicine, University “Ovidius” Constanţa, Romania

\begin{abstract}
.
Our results were obtained on a total of 101 cases and the presence of the colosigmoid trunk was encountered in $54.46 \%$ of cases, with seven types of branching variation. We were able to compare our results with those of Lippert, noticing that the classical anatomy only mentions (without details) the presence of the colosigmoid trunk, while other authors do not even mention it. The anatomical variations of the colosigmoid arterial trunk are: 1 . from the colosigmoid trunk originate the left colic artery and a sigmoid trunk that gives origin to all three sigmoid arteries ( $14.85 \%$ of cases); 2 . from the colosigmoid trunk originate the left colic and the superior sigmoid arteries (18.81\% of cases); 3 . from the colosigmoid trunk originate the left colic artery and a sigmoid trunk that give origin to two sigmoid arteries, superior and inferior, an aspect found in $4.95 \%$ of cases; 4. a colosigmoid trunk that give rise to the inferior sigmoid artery and to a lower trunk that give the left colic artery and the superior sigmoid artery (5.94\% of cases); 5. a colosigmoid trunk that give origin to the left colic, middle colic and superior sigmoid arteries in $5.94 \%$ of cases; 6 . a colosigmoid trunk that splits into a colic branch which further gives origin to the left and middle colic arteries and into a sigmoid branch that will give the superior and inferior sigmoid arteries (1.98\% of cases); 7. a colosigmoid trunk that gives origin to the middle colic artery (for the transverse colon), to the left colic and superior sigmoid arteries (1.98\% of cases).
\end{abstract}

Iliescu D.M.

Department of Anatomy, Faculty of medicine, University "Ovidius" Constanţa, Romania

Aleea Universitatii, Nr. 1, Campus B

Constanţa, Romania

dan@anatomie.ro

\section{Introduction}

The study of the inferior mesenteric vessels within the literature expands over more than four centuries while the studies of the inferior mesenteric artery are much larger than the ones regarding the inferior mesenteric vein. The findings that were published are sometimes very different even when using identical research methods. When a surgeon operates on the large intestine, to a relatively large resection that includes a voluminous artery, he often faces anatomic vascular variations, sometimes different from those described in standard textbooks. We notice a very large number of anatomists that have contributed to the study of mesenteric vessels, especially the German and the French schools that particularly distinguished. Their careful dissection and their observations were made with great effort, although every author performed on a limited number of anatomical samples. In fact, these findings often disagreed with the predecessor's results, also the result of research findings on a limited number of cases. Later, the surgeons completed the anatomists, because they had access to new ways of visualization and a rich clinical material for study; radiologists followed them. However, while the methodology used by radiologists, as selective arteriography, provides highly accurate anatomical details, the surgeon can monitor and control the vascular network beyond the limits allowed by the operator field. Moreover, the radiological representation of vascular anatomy is the only bi- or tri-dimensional analysis, despite the modern equipment. I believe that our research can help surgeons and radiologists among the variety of origin 
of inferior mesenteric artery collateral branches, most common being the colosigmoid trunk. Our research shows the necessity for the surgeon to know the distribution of colic vessels of the patient, before it is subjected to a large colon resection, especially if restoration of continuity is required.

\section{Material and methods}

We performed our study on 101 cases, assessing the origin and the mode of termination of the collateral branches of the inferior mesenteric artery. We work on human cadavers, adult and fetal, as well as on organic subdiaphragmatic blocks, using as study methods the dissection and the plastic injection followed by corrosion or dissection. We also evaluated 68 abdominal angiographies, simple and angioCT.

\section{Results and discussions}

The presence of a colosigmoid trunk was encountered in 55 cases ( $54.46 \%$ of cases), describing 7 variants of branching. The trunk name presumes that in each of its variants it gave origin to the left colic artery, thus varying the number of other possible colic and sigmoid arteries. We could compare our results with those of Lippert [1], as long as classical anatomists such as Paturet [2] or Rouvière [3] just mention the presence of the colosigmoid trunk, without variants and indicating the possibility of origin only for the superior sigmoid artery from this trunk. Other authors such as Testut [4], Godlewski [5] or Kamina [6,7] does not even mention the existence of this arterial trunk.
Variations of the colosigmoid arterial trunk

Type 1 - from the colosigmoid trunk originate the left colic artery and a sigmoid trunk that gives birth to all three sigmoid arteries, in 15 cases $(14.85 \%$ of cases) (Figure 1);

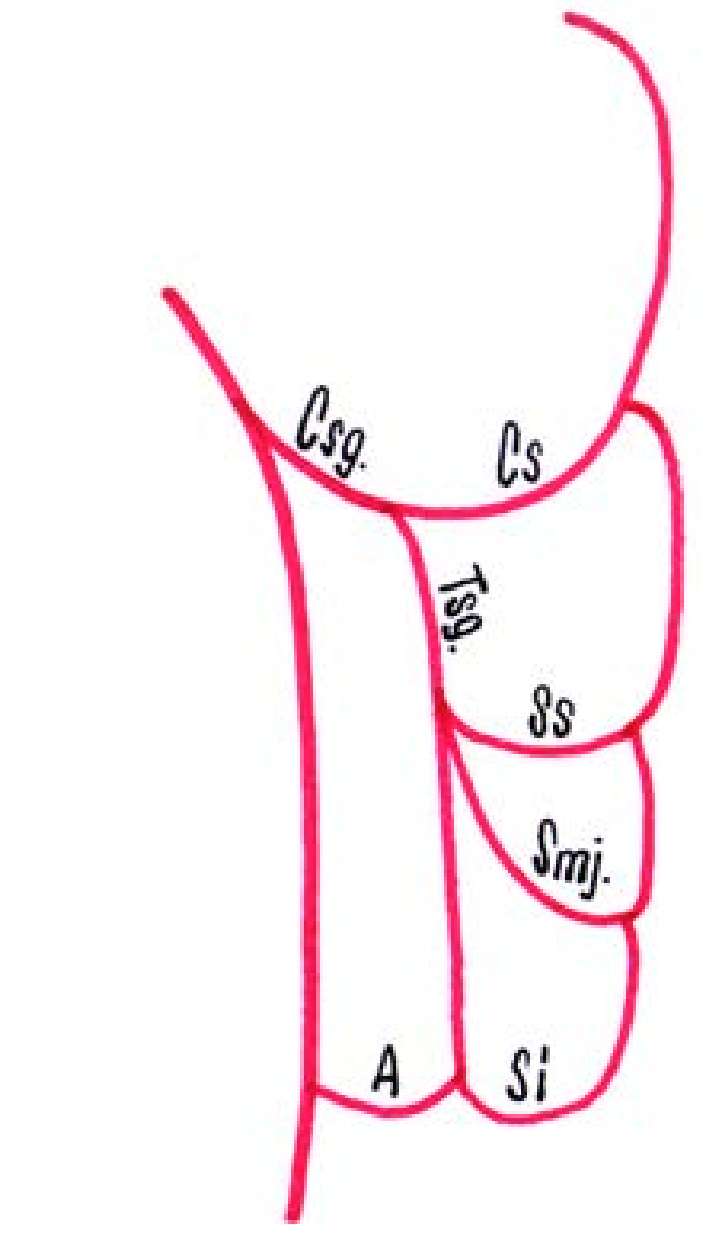

Figure 1 - Schema of type 1: Im: inferior mesenteric; Csg: colosigmoid trunk; Cs: left colic; Tsg: sigmoid trunk; Ss: superior sigmoid; Smj: middle sigmoid; Si: inferior sigmoid; A: recto-sigmoid anastomosis; Rs: superior rectal

Type 2 - from the colosigmoid trunk originate the left colic and the superior sigmoid arteries, in 19 cases (18.81\% of cases) (Figures 2,3); [1] encountered this type in $30 \%$ of cases; 


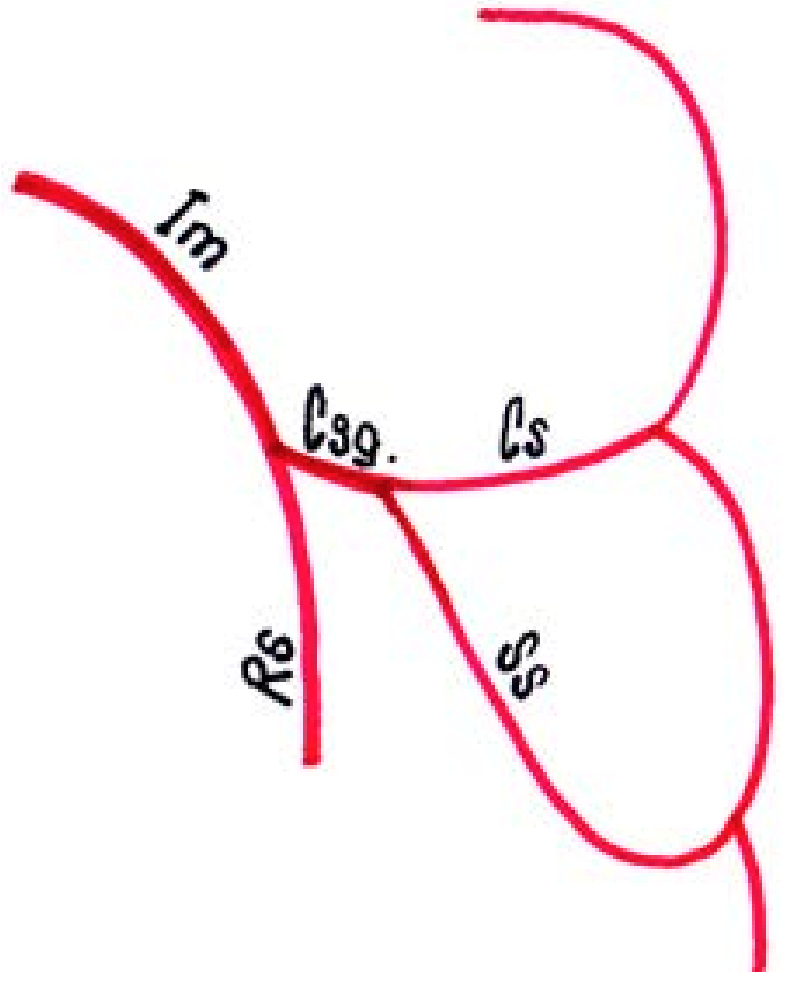

Figure 2 - Schema of type 2: Im: inferior mesenteric; Csg. colosigmoid trunk; Cs: left colic; Ss: superior sigmoid; Rs: superior rectal.

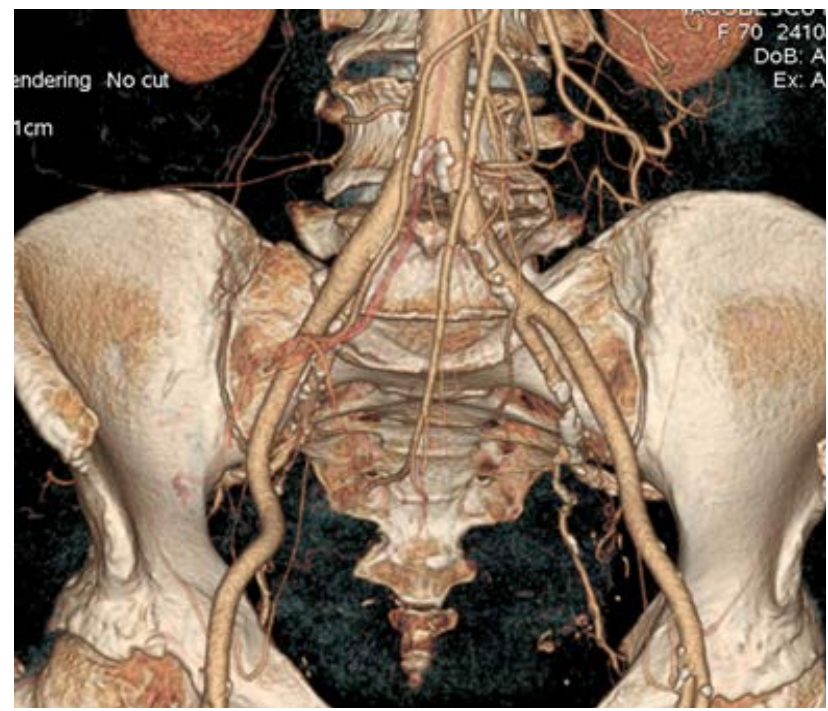

Figure 3 - Type 2: left colic and the superior sigmoid arteries from the colosigmoid trunk
Type 3 - from the colosigmoid trunk originate the left colic artery and a sigmoid trunk that give origin to two sigmoid arteries, superior and inferior, an aspect found in 5 cases $(4.95 \%$ of cases) (Figure $4)$;

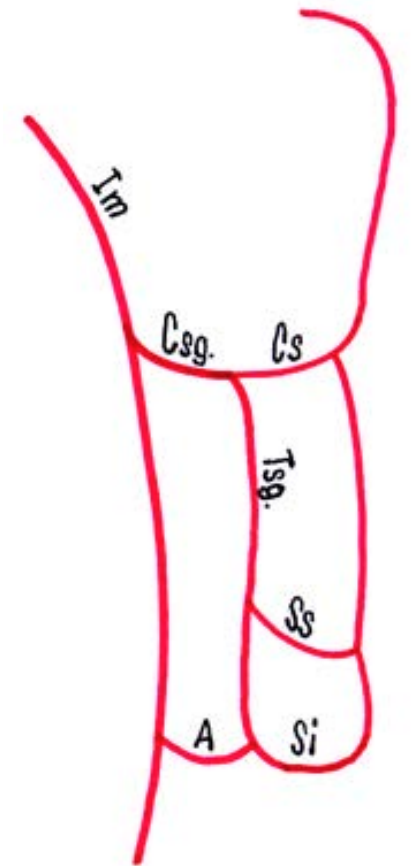

Figure 4 - Schema of type 3: Im: inferior mesenteric; Csg: colosigmoid trunk; Cs: left colic; Tsg: sigmoid trunk; Ss: superior sigmoid; Si: inferior sigmoid; A: recto-sigmoid anastomosis; Rs: superior rectal

Type 4 - a colosigmoid trunk that gives rise to the inferior sigmoid artery and to a lower trunk that give the left colic artery and the superior sigmoid artery in 6 cases $(5.94 \%$ of cases 0 (Figures 5, 6); [1] encountered this type in $9 \%$ of cases; 


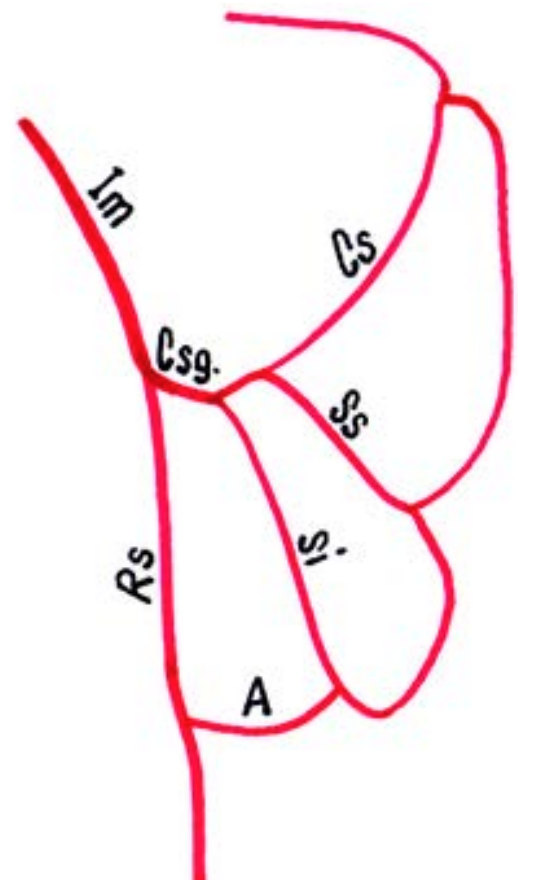

Figure 5 - Schema of type 4: Im: inferior mesenteric; Csg: colosigmoid trunk; Cs: left colic; Ss: superior sigmoid; Si: inferior sigmoid; A: recto-sigmoid anastomosis; Rs: superior rectal

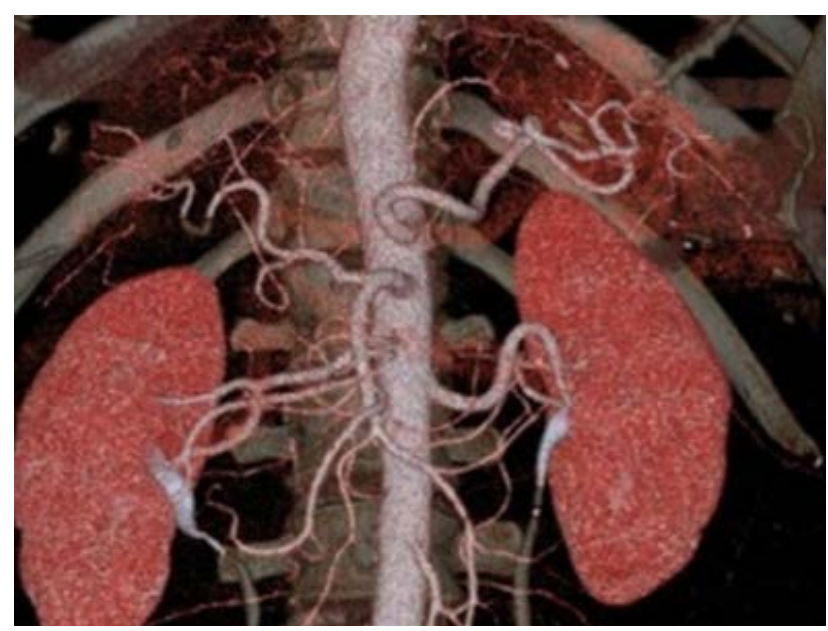

Figure 6 - Type 4

Type 5 - a colosigmoid trunk that give origin to the left colic, middle colic and superior sigmoid arteries in 6 cases (5.94\% of cases) (Figure 7);

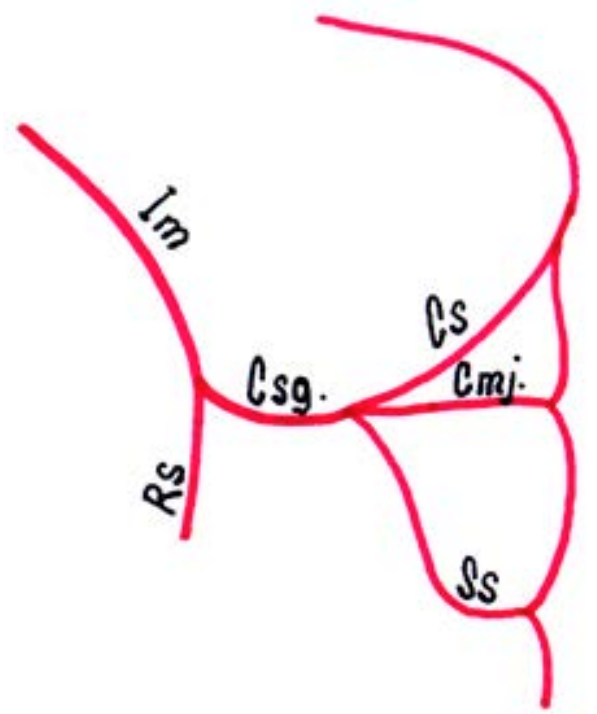

Figure 7 - Schema of type 5: Im: inferior mesenteric; Csg: colosigmoid trunk; Cs: left colic; Cmj: middle colic; Ss: superior sigmoid; Rs: superior rectal

Type 6 - a colosigmoid trunk that splits into a colic branch which further gives origin to the left and middle colic arteries and into a sigmoid branch that will give the superior and inferior sigmoid arteries in 2 cases (1.98\% of cases) (Figures 8,9$)$;

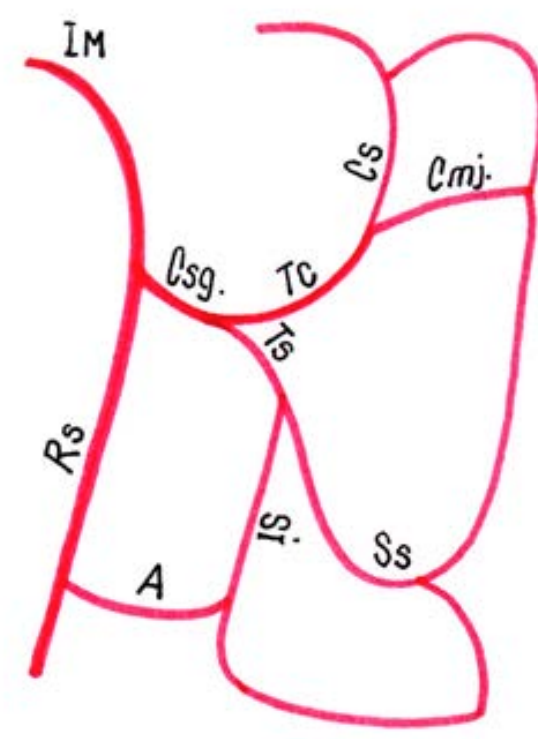

Figure 8 - Schema of type 6: Im: inferior mesenteric; Csg: colosigmoid trunk; Cs: left colic; Cmj: middle colic; $T s$ : sigmoid trunk; Ss: superior sigmoid; Si: inferior sigmoid; Rs: superior rectal; A: recto-sigmoid anastomosis 


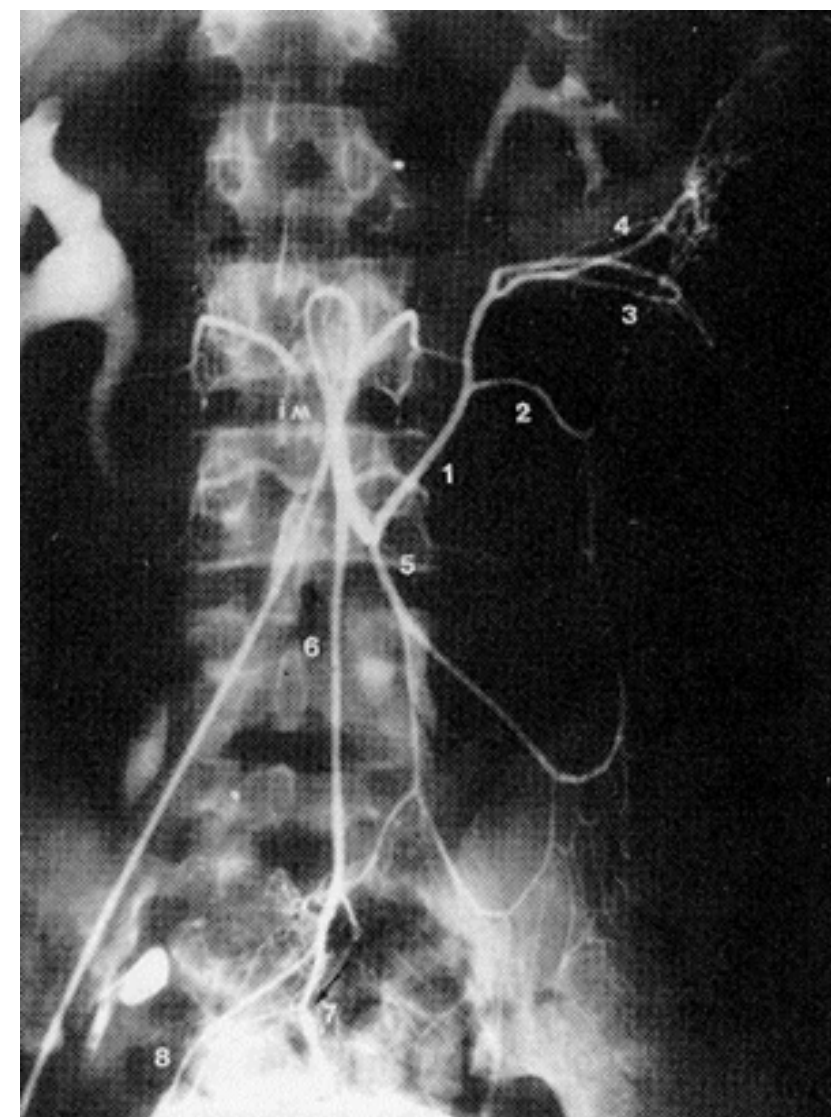

Figure 9 - The left colic artery originates from a colosigmoid trunk that splits into a colic branch which further gives origin to the left and middle colic arteries and into a sigmoid branch that will give the superior and inferior sigmoid arteries; Im: inferior mesenteric; 1. colic trunk; 2. middle colic; 3-4. left colic and its branches; 5 . sigmoid trunk; 6. sup. rectal a.; 7-8. bifurcating branches of the superior rectal a.; 9. superior sigmoid a.; 10. inferior sigmoid a.

Type 7 - a colosigmoid trunk that gives origin to the middle colic artery (for the transverse colon), to the left colic and superior sigmoid arteries, in 2 cases (1.98\% of cases) (Figure 10); [1] encountered this type in $5 \%$ of cases;

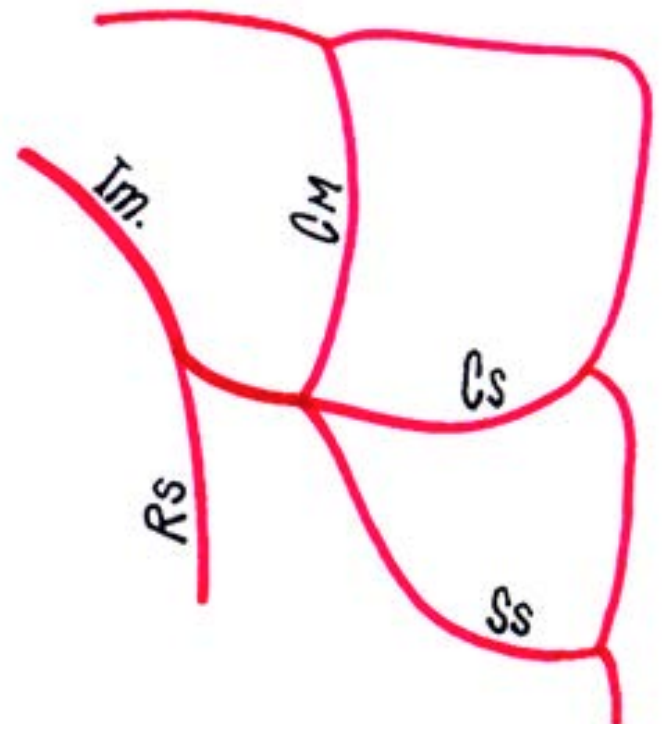

Figure 10 - Schema of type 7: Im: inferior mesenteric; Cmj: middle colic; Cs: left colic; Ss: superior sigmoid; Rs: superior rectal

\section{Conclusions}

The high frequency of the presence of the colosigmoid trunk with its variants occurs mostly in cases when the inferior mesenteric artery is shorter, especially in the first variant, when the superior rectal artery has a high origin; a similar aspect is encountered when from the colosigmoid trunk start two sigmoid arteries, with two variants: the two arteries are the only sigmoid arteries, or they may be the superior and the middle sigmoid arteries, while the third and (rarely) the fourth start from the inferior mesenteric artery, under the origin of the colosigmoid trunk.

In the other 46 cases ( $45.54 \%$ of cases), we met two variations:

- the existence of a colic trunk, an aspect encountered in 7 cases $(6.03 \%$ of cases), with two or even three colic arteries;

- in 39 cases $(38.61 \%$ of cases) the sigmoid arteries started under the origin of the colosigmoid or 
of the trunk colic.

The branches arising from the colosigmoid trunk are important in assessing the extent of the descending colic vascular territory and the superior rectal artery origin.

\section{References}

1. Lippert, H. \& Pabst, R. (1985). Arterial variations in man. Classification and Freqvency. (pp. 4851). Munchen, Germany: JF Bergman Verlag.

2. Paturet, G. (1964). Traite d'anatomie humaine: Appareil circulatoire. Tome III, Fasc I. (pp. 489510). Paris: Masson.

3. Rouvière, H. \& Delmas, A. (1997). Anatomie Humaine déscriptive topographique et fonctionnelle. Tome 2, 14-edition, (pp. 185-190, 398, 428). Paris: Masson.
4. Testut, L. (1924). Traité d'Anatomie humaine. Tome deuxième. Angéiologie. (pp. 524-526). Paris: Ed. Gaston Doin.

5. Godlewski, G. (1994). Le colon. In: Chevrel J.P. (Ed.), Anatomie clinique. Le tronc (pp. 363). Paris: Ed. Springer-Verlag.

6. Kamina, P. \& Di Marino, V. (1997). Abdomen. Parois et Appareil digestif. Tome 1, (pp. 114-116, 126, 147-148). Paris: Ed. Maloine.

7. Kamina, P. \& Di Marino, V. (1998). Abdomen. Appareil digestif et rein. Tome 2, (pp. 31-38). Paris: Ed. Maloine.

8. $* * * * *$ Terminologia Anatomica. International Anatomical Terminology (1988). (pp. 8788). Federative Committee on Anatomical Terminology. Stuttgart: Thieme Verlag. 